\title{
OPTIMIZATION OF A DISC-TYPE MAGNETO-RHEOLOGICAL CLUTCH
}

\author{
HORVÁTH P., TÖRŐCSIK D.
}

\author{
Széchenyi István University, Egyetem tér 1, \\ Győr, 9026, Hungary
}

\begin{abstract}
In the last decade magnetorheological (MR) clutches gained high attention because of their beneficial properties. One of the most important issues is to find ways to increase their transmissible torque. This paper summarizes first the basics of magnetorheology and focuses later on the investigation on the optimal inner radius of a MR clutch. Both a simple analytical method and a simulation procedure is presented.
\end{abstract}

KEY WORDS: Magnetorheological fluid, shear stress, torque, saturation, simulation

\section{INTRODUCTION}

Magnetorheological fluid (MRF) was first applied in shock absorbers, vibration isolation devices and semi active car suspensions. Nowadays high efforts are taken to use it in brakes and clutches. The main stream of ongoing research focuses on increasing the transmittable torque of these devices so that they can gain real industrial application. The most important part of all magnetorheological devices is the magnetorheological fluid itself. It is to be noted that its relative permeability is less than 6, consequently MRF presents high reluctance in the magnetic circuit. The fluid contains carbonyl iron powder, which is suspended in oil with additives. The major role of using additives is to prevent the precipitation of the iron powder in the suspension. Diameters of the iron powder particles are 1-10 $\mu \mathrm{m}$ in size. In the absence of an applied magnetic field the particles are in disordered position in the fluid. With the application of a magnetic field the iron particles become polarized and form chains in the direction of the flux lines. The enhancement of the magnetic field modifies the apparent viscosity of the fluid. The shear stress occurring in the fluid when loaded under magnetization can be calculated by the following formula:

$$
\tau=\tau(B)+\eta \frac{d v}{d y}
$$

where

$\tau \quad$ shear stress depending on the magnetic field

$\eta \quad$ dynamic viscosity of the fluid

$\mathrm{dv} / \mathrm{dy} \quad$ speed gradient in the gap.

The second term of the formula originates from the Newtonian behavior of the fluid and it is generally small relative to the first one. Typical shear stress curve of commercially available MR 132 LD magnetorheological fluid is published by the supplier LORD Corp. [1] and it can be seen in Fig. 1. 


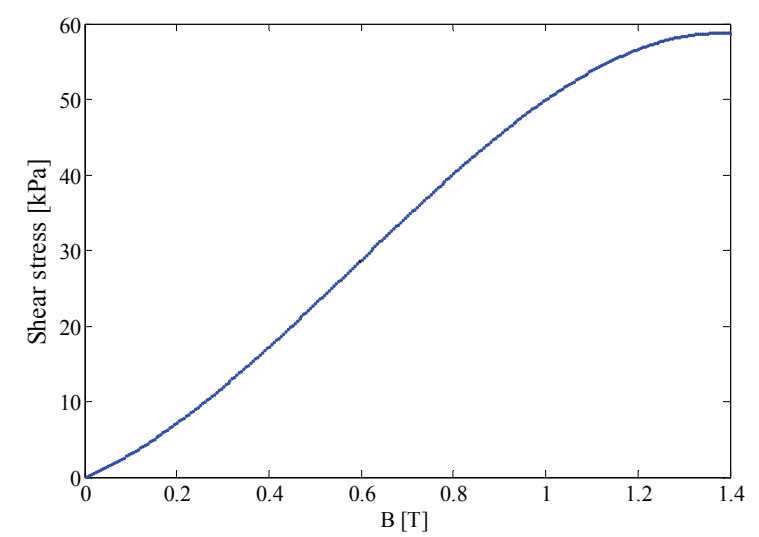

Fig. 1 Shear stress of MRF vs. magnetic flux density

In the last decade one can find many works in literature dealing with MRF applications in clutches. The reason for this can be summarized as follows: little wea due to the simple construction without moving parts and direct controllability with very short time constant. Unfortunately there are also some shortcomings such as moderate transmissible torque, non-negligible energy consumption as well as ageing of MRF. Bansbach [2] suggested a double-plate and a multi-plate MRF clutch to be placed between the car engine and the differential. Lampe [3] studied the transitional state of a brake between liquid and solid states. Kavlicoglu [4] at the University of Nevada a prototype double-plate MRF clutch of 7,9 Nm torque was built. Later their group increased the torque to 240 $\mathrm{Nm}$ applying 43 plates. Gratzer et al. at MAGNA [5] prepared a multi-cylindrical MRF clutch of $700 \mathrm{Nm}$ torque with a magnetization unit rotating together with the shaft. Its current was supplied with slip rings.

One can distinguish disc-type and cylindrical MR clutches. Our work deals only with the former one (Fig.2). The gaps between driving (1) and driven parts (2) are filled with MRF. introducing electricity in to the coil (3) of the magnetization unit (4), the apparent viscosity of the MRF changes due to the electromagnetic field. Trying to rotate driving and driven shafts relative to each other, stretching particle chains of MRF exert shear stress between adjacent surfaces.

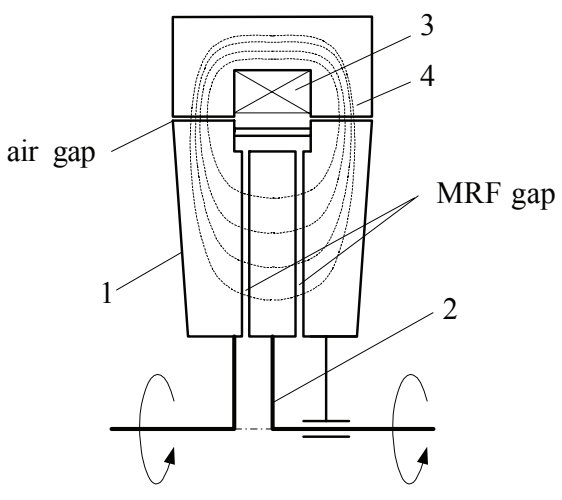

Fig. 2: MR clutch 
Torque due to shear stress acting on a circular plate of inner radius $r_{0}$ and outer radius $r_{1}$ can be calculated with the equation:

$$
\mathrm{T}=2 \pi \int_{\mathrm{r}_{0}}^{\mathrm{r}_{1}} \tau(\mathrm{B}) \mathrm{r}^{2} \mathrm{dr}
$$

In practice one of the most important issues is how to increase the transmissible torque. Studying equation (2) two ways seem to be possible. First, one can achieve optimal B(r) magnetic flux density distribution along the radius. Second, one has to apply the correct $r_{0} / r_{1}$ ratio of the plate. Both propositions must be realized at a given magnetization power, characterized principally by magnetic flux $\Phi_{0}$. The former possibility had been investigated in [6] and conclusion were drawn that nearly constant magnetic flux density along radius can be regarded optimal and easy to realize especially in the case of multi-plate clutches.

This paper focuses on determining the optimal inner radius of the plate. First a simplified analytical calculation is presented and after that its findings are compared by numerical simulation.

\section{ANALYTICAL METHOD}

For a simple analytical calculation of magnetic circuit some assumptions are to be satisfied. There must not be sharp changes in cross-section area as well as the thickness of all gaps must be small. If these assumptions are obtained, magnetic field density can be regarded as constant in any cross-section. In the case of a circular plate of inner radius $r_{0}$ and outer radius $r_{1}$ the mean value of the theoretical magnetic flux density under given magnetic flux $\Phi_{0}$ is

$$
\overline{\mathrm{B}}_{\mathrm{t}}=\frac{\Phi_{0}}{\left(\mathrm{r}_{1}^{2}-\mathrm{r}_{0}^{2}\right) \pi}
$$

It is to be taken into account that as $r_{0}$ approaches $r_{1}$, theoretically calculated magnetic flux density is no longer valid because of the saturation of the flux guide parts and magnetorheological fluid (Fig.3).

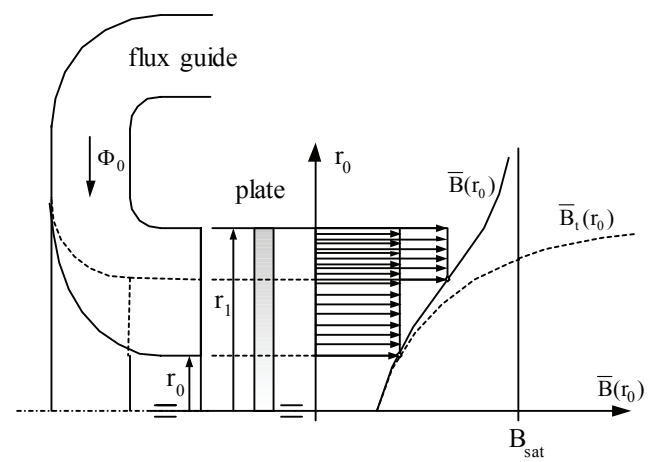

Fig.3 Theoretical magnetic flux density vs. inner radius 
Higher values then the saturation limit $\mathrm{B}_{\mathrm{s}}$ of the steel cannot evolve. Saturation can be taken into account in the knowledge of $\mathrm{B}(\mathrm{H})$ curve of the applied ferromagnetic low carbon steel $\mathrm{C} 10$ (AISI 1010) by the simplified formula

$$
\mathrm{B}=\left\{\begin{array}{l}
\mathrm{B}_{t} \text { if } \mathrm{B}_{t}<1.6 \mathrm{~T} \\
1.6 \mathrm{~T} \quad \text { else }
\end{array}\right.
$$

For analytical investigation shear stress variation presented in Fig. 1 can be approximated by the

$$
\tau \approx-31.2 \mathrm{~B}^{3}+54.8 \mathrm{~B}^{2}+26.4 \mathrm{~B}
$$

third order equation where $\tau$ is measured in $\mathrm{kPa}$. Torque exerted by the MRF on a circular disc can be calculated after substitution (3), (4) and (5) expressions into (2). The result of the analytical investigation is depicted in Fig. 4 with continual line.

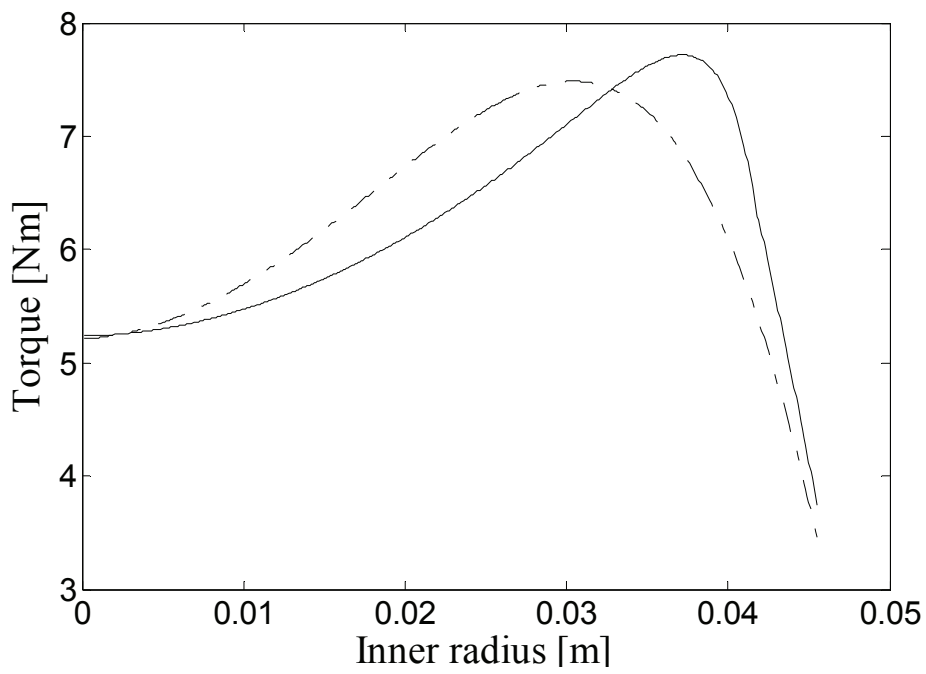

Fig.4 Transmissible torque vs. inner radius curve

As suspected before there exists really an optimal value of the inner radius. If the plate is manufactured with inner radius $\mathrm{r}_{0} \approx 0,74 \mathrm{r}_{1}$ transmissible torque has a sharp maximum.

\section{SIMULATION OF THE MAGNETIC CIRCUIT}

To check our findings we carried out simulations by COMSOL Multiphysics software on the same configuration. In Fig. 5 one can see the relevant parts of the magnetic circuit where the darkness of picture is proportional to the intensity of magnetic flux density. Assuming $J=4 \cdot 10^{6}$ $\mathrm{A} / \mathrm{m}^{2}$ constant magnetization current density and $h=1 \mathrm{~mm}$ fluid gap thickness, magnetic flux density was computed along the MRF gap assuming an inner radii of 16, 20, 24, 28 and $32 \mathrm{~mm}$, respectively. 


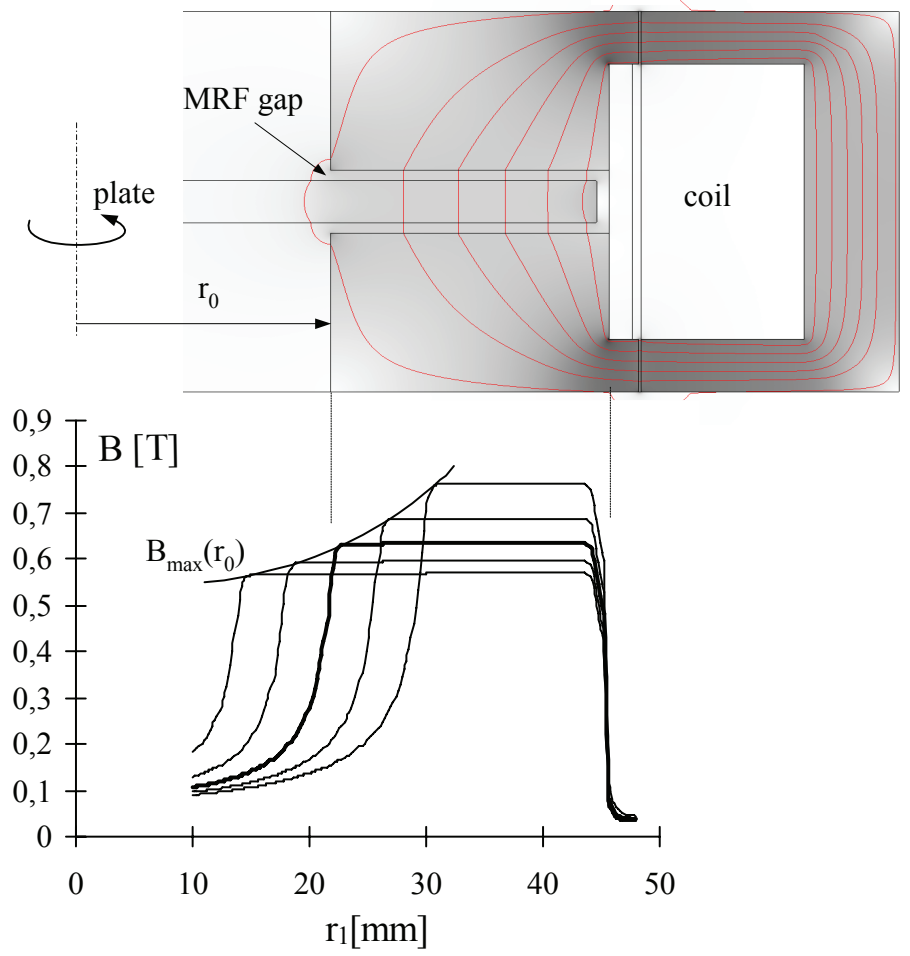

Fig.5 Magnetic flux density variation along the radius

As one can see in Fig. 5 the simulation proved that magnetic flux density was almost constant along the radius as it had been assumed in the analytical investigation. The equation of the envelope $\mathrm{B}_{\max }\left(\mathrm{r}_{0}\right)$ laid on the corner points of the graph can be approximated by the

$$
B_{\max }\left(r_{0}\right)=300 r_{0}^{2}-0.055 r_{0}+0.446[\mathrm{~T}]
$$

second order regression function. Equation (6) gained by simulation is the counterpart of equation (3) of analytical solution. The relationship between the torque transmitted and inner radius of the plate gained by simulation is depicted with a dotted line in Fig. 4. According to the more accurate simulation procedure the optimal inner radius of plate happened at $r_{0} \approx 0.6 r_{1}$. The reason for the difference between graphs can originate in many things. Analytical method can consider neither real geometry of flux guides, nor real material properties. It neglects also the resistance of MRF. The only advantage is the fast and globally appropriate solution. If accurate results are needed, the simulation procedure is firmly recommended.

\section{CONCLUSIONS}

This paper presented an analytical and a numerical method to calculate the optimal inner radius of a disc-type magnetorheological clutch. It turned out that transmissible torque has its 
maximum near $\mathrm{r}_{0} / \mathrm{r}_{1}=0.6 \ldots 0.74$ ratio, assuming constant magnetic flux density along the plate's radius. The result of the analytical calculation has been checked with numerical simulation. Owing to the remarkable difference between their results, simulation procedure is recommended instead of analytical calculations. Another interesting result of the investigation is that transmissible torque exerted by a MR layer of outer radius $r_{1}=0.05 \mathrm{~m}$ is only about $7-8 \mathrm{Nm}$. For industrial applications a multi-plate construction seems to be favorable.

\section{ACKNOWLEDGEMENT}

Our research was supported by the project „TAMOP-4.2.1/B-09/1/KONV-2010-0003: Mobility and Environment: Research in the fields of motor vehicle industry, energetics and environment in the Central- and Western-Transdanubian Regions of Hungary". The Project is supported by the European Union and co-financed by the European Social Fund.

\section{REFERENCES}

[1] www.lord.com/Products-and-Solutions

[2] BANSBACH, E. E.: Torque Transfer Apparatus Using Magnetorheological Fluids. US Patent, No: 5,779,013, 1998.

[3] LAMPE, D.: Anwendung von Magnetorheologischen Fluiden in Kupplungen, www.donnerflug.de/publikationen/antrieb.pdf

[4] B.M. KAVLICOGU; F. GORDANINEJAD; C.A. EVRENSEL; N.COBANOGLU; Y. Liu, A. FUCHS.: A high-torque magneto-rheological fluid clutch. Proceedings of SPIE Conference on Smart Materials and Structures, San Diego, 2002.

[5] GRATZER, F.; STEINWENDER, H.; KUSEJ, A.: Magnetorheologische Allradkupplungen. ATZ, Jahrgang 10, 2008. p. 902-909

[6] HORVÁTH, P.; TÖRÖCSIK, D.: Optimization of MR clutches. Proceedings of Machine Design Conference. Miskolc, 2011. 